Pacific

Journal of

Mathematics

GLOBAL WELL-POSEDNESS FOR THE 3D ROTATING NAVIER-STOKES EQUATIONS WITH HIGHLY OSCILLATING INITIAL DATA

Qionglei Chen, ChangXing MiaO and Zhifei Zhang 


\title{
GLOBAL WELL-POSEDNESS FOR THE 3D ROTATING NAVIER-STOKES EQUATIONS WITH HIGHLY OSCILLATING INITIAL DATA
}

\author{
Qionglei Chen, ChangXing MiaO And Zhifei Zhang
}

We prove the global well-posedness for the 3D rotating Navier-Stokes equations in the critical functional framework. This result allows us to construct global solutions for a class of highly oscillating initial data.

\section{Introduction}

In this paper, we study the 3D rotating Navier-Stokes equations

$$
\left\{\begin{array}{l}
u_{t}-v \Delta u+\Omega e_{3} \times u+u \cdot \nabla u+\nabla p=0 \\
\operatorname{div} u=0 \\
u(0, x)=u_{0}(x)
\end{array}\right.
$$

where $v$ denotes the viscosity coefficient of the fluid, $\Omega$ the speed of rotation, $e_{3}$ the unit vector in the $x_{3}$ direction and $\Omega e_{3} \times u$ the Coriolis force. We refer to [Chemin et al. 2006; Majda 2003; Pedlosky 1987] for its background in geophysical fluid dynamics. If the Coriolis force is neglected, the equations (1-1) become the classical 3D incompressible Navier-Stokes equations

$$
\left\{\begin{array}{l}
u_{t}-v \Delta u+u \cdot \nabla u+\nabla p=0 \\
\operatorname{div} u=0 \\
u(0, x)=u_{0}(x)
\end{array}\right.
$$

The global existence of a weak solution of (1-1) can be proved by the classical compactness method, since we still have the energy estimate

$$
\|u(t)\|_{L^{2}}^{2}+2 \int_{0}^{t}\|\nabla u(s)\|_{L^{2}}^{2} d s \leq\left\|u_{0}\right\|_{L^{2}}^{2} .
$$

As in 3D Navier-Stokes equations, the uniqueness and regularity of weak solutions are also open problems. Recently, Giga et al. [2006; 2007; 2008] studied the local

Chen is supported by NSF of China (NSFC) grants 10701012 and 10931001 . Miao is supported by NSFC grants 11171033 and 11231006. Zhang is supported by NSFC grant 10990013.

MSC2000: primary 35Q30; secondary 35D10.

Keywords: rotating Navier-Stokes equations, global well-posedness, Besov space, highly oscillating. 
existence of a mild solution for a class of nondecaying initial data which includes a class of almost periodic functions, as well as global existence for small data. On the other hand, when the speed $\Omega$ of rotation is fast enough, the global existence of smooth solution was proved in [Babin et al. 1997; 1999; Chemin et al. 2000; 2006].

For the 3D Navier-Stokes equations, Fujita and Kato [1964; Kato 1984] proved the local well-posedness for large initial data and the global well-posedness for small initial data in the homogeneous Sobolev space $\dot{H}^{\frac{1}{2}}$ and the Lebesgue space $L^{3}$, respectively. These spaces are all the critical ones, which are relevant to the scaling of the Navier-Stokes equations: if $(u, p)$ solves (1-2), then

$$
\left(u_{\lambda}(t, x), p_{\lambda}(t, x)\right):=\left(\lambda u\left(\lambda^{2} t, \lambda x\right), \lambda^{2} p\left(\lambda^{2} t, \lambda x\right)\right)
$$

is also a solution of (1-2). The so-called critical space is the one such that the associated norm is invariant under the scaling of (1-3). Recently, Cannone [1997] (see also [Cannone 1995; 2004; Cannone et al. 1994]) generalized it to Besov spaces with negative index of regularity. More precisely, he showed that if the initial data satisfies

$$
\left\|u_{0}\right\|_{\dot{B}_{p, \infty}^{-1+\frac{3}{p}}} \leq c, \quad p>3
$$

for some small constant $c$, then the Navier-Stokes equations (1-2) are globally well-posed. Let us emphasize that this result allows us to construct global solutions for highly oscillating initial data which may have a large norm in $\dot{H}^{\frac{1}{2}}$ or $L^{3}$. A typical example is

$$
u_{0}(x)=\sin \frac{x_{3}}{\varepsilon}\left(-\partial_{2} \phi(x), \partial_{1} \phi(x), 0\right)
$$

where $\phi \in \mathscr{Y}\left(\mathbb{R}^{3}\right)$ and $\varepsilon>0$ is small enough. We refer to [Chemin and Gallagher 2006; Chemin and Zhang 2007; Chen et al. 2010a] for some relevant results. A natural question is then to prove a theorem of this type for the rotating Navier-Stokes equations.

We know that Kato's method heavily relies on the uniform boundedness of the Stokes semigroup in $L^{p}$ and global $L^{p}-L^{q}$ estimates, but the Stokes-Coriolis semigroup is not uniformly bounded in $L^{p}$ for $p \neq 2$; see Theorems 5 and 6 in [Dragičević et al. 2006]. Standard techniques allow us to prove these estimates only locally for the Stokes-Coriolis semigroup, hence one can obtain the local existence of mild solution in $L^{3}$ by Kato's method. Whether one can extend this solution to a global one for small data in $L^{3}$ is a very interesting problem.

Very recently, based on the global $L^{p}-L^{q}$ estimates with $q \leq 2 \leq p$ and $L^{q}-H^{\frac{1}{2}}$ estimates with $q>3$ for the Stokes-Coriolis semigroup, Hieber and Shibata [2010] proved the following global result for small data in $H^{\frac{1}{2}}$. 
Theorem 1.1. Let $q>3$. Then there exists $c>0$ independent of $\Omega$ such that for any $u_{0} \in H_{\sigma}^{\frac{1}{2}}$ with $\left\|u_{0}\right\|_{H^{\frac{1}{2}}} \leq c$, the equations (1-1) admit a unique mild solution $u \in C\left([0, \infty), H_{\sigma}^{\frac{1}{2}}\right)$ satisfying

$$
\begin{gathered}
u \in C\left((0, \infty), L^{q}\right) \quad \text { and } \quad \lim _{t \rightarrow 0^{+}} \sup _{0<s<t} s^{\frac{1}{2}-\frac{3}{2 q}}\|u(s, \cdot)\|_{L^{q}}=0, \\
\nabla u \in C\left((0, \infty), L^{2}\right) \quad \text { and } \quad \lim _{t \rightarrow 0^{+}} \sup _{0<s<t} s^{\frac{1}{4}}\|\nabla u(s, \cdot)\|_{L^{2}}=0 .
\end{gathered}
$$

Here $H_{\sigma}^{\frac{1}{2}}$ denotes the closure of the set $\left\{u \in C_{c}^{\infty}\left(\mathbb{R}^{3}\right)^{3}, \operatorname{div} u=0\right\}$ in the norm of $\|\cdot\|_{H \frac{1}{2}}$.

The goal of this paper is to prove the global existence of a solution of (1-1) for a class of highly oscillating initial velocity. Thus we need to solve the system (1-1) for the initial data in a critical functional framework whose regularity index is negative, for example, $\dot{B}_{p, q}^{-1+\frac{3}{p}}$ for $p>3$. However, Cannone's proof [1997] doesn't work for our case, since it also relies on the global $L^{p}-L^{q}$ estimates for the Stokes semigroup. Indeed, for the Stokes-Coriolis semigroup $\mathscr{G}(t)$, one has

$$
\left\|\mathscr{G}(t) u_{0}\right\|_{L^{p}} \leq C_{p, \Omega} t^{2}\left\|u_{0}\right\|_{L^{p}}, \quad \text { if } p \neq 2 ;
$$

see Proposition 2.2 in [Hieber and Shibata 2010]. Then we can infer from the definition of the Besov space that

$$
\left\|\mathscr{G}(t) u_{0}\right\|_{\dot{B}_{p, q}^{-1+\frac{3}{p}}} \leq C t^{2}\left\|u_{0}\right\|_{\dot{B}_{p, q}^{-1+\frac{3}{p}}} .
$$

This means that even if the initial data $u_{0}$ is small in $\dot{B}_{p, q}^{-1+\frac{3}{p}}$, the linear part of the solution, $\left\|\mathscr{G}(t) u_{0}\right\|_{\dot{B}_{p, q}^{-1+\frac{3}{p}}}$, may become large after some time $t_{0}>0$.

Fortunately, we have the following important observation: if $u$ is an element of $L^{p}$ with supp $\hat{u} \in\{\xi:|\xi| \gtrsim \lambda\}$, then

$$
\|\mathscr{G}(t) u\|_{L^{p}} \leq C_{p, \Omega} e^{-t \lambda^{2}}\|u\|_{L^{p}}
$$

for any $p \in[1, \infty]$ and $t \in[0, \infty]$, while for any $u \in L^{2}$,

$$
\|\mathscr{G}(t) u\|_{L^{2}} \leq\|u\|_{L^{2}} .
$$

This motivates us to introduce the hybrid-Besov spaces $\dot{\mathscr{B}}_{2}^{\frac{1}{2}, \frac{3}{p}-1}$ (see Definition 2.2). Roughly speaking, if $u \in \dot{\mathscr{B}}_{2}^{\frac{1}{2}, p}, \frac{3}{p}-1$, the low frequency part of $u$ belongs to $\dot{H}^{\frac{1}{2}}$ and the high frequency part belongs to $\dot{B}_{p, \infty}^{-1+\frac{3}{p}}$. So, $\dot{\mathscr{S}}_{2, p}^{\frac{1}{2}}, \frac{3}{p}-1$ is still a critical space. A remarkable property of $\dot{\mathscr{S}}_{2, p}^{\frac{1}{2}, \frac{3}{p}-1}$ is that if $p>3$, then

$$
\left\|u_{0}(x)\right\|_{\dot{\mathscr{B}}_{2, p} \frac{1}{2}, \frac{3}{p}-1} \leq C \varepsilon^{1-\frac{3}{p}}
$$


for $u_{0}(x)=\sin \left(x_{1} / \varepsilon\right) \phi(x)$, with $\phi(x) \in \mathscr{Y}\left(\mathbb{R}^{3}\right)$; see Proposition 2.4. That is, the highly oscillating function is still small in the norm of $\dot{\mathscr{B}}_{2} \frac{1}{2}, \frac{3}{p}-1$.

Definition 1.2. Let $1 \leq p \leq \infty$, we denote by $E_{p}$ the space of functions such that

$$
E_{p}=\left\{u: \operatorname{div} u=0,\|u\|_{E_{p}}<+\infty\right\},
$$

where

$$
\|u\|_{E_{p}}:=\|u\|_{\widetilde{L}^{\infty}\left(\mathbb{R}^{+} ; \dot{\mathscr{B}}_{2, p}^{\frac{1}{2}, \frac{3}{p}-1}\right)}+\|u\|_{\widetilde{L}^{1}\left(\mathbb{R}^{+} ; \dot{\Re}_{2, p}^{\frac{5}{2}, \frac{3}{p}+1}\right)} .
$$

Definition 1.3. We denote by $C_{*}\left([0, \infty) ; \dot{\mathscr{P}}_{2} \frac{1}{2}, \frac{3}{p}-1\right)$ the set of functions $u$ such that $u$ is continuous from $(0, \infty)$ to $\dot{\mathscr{B}}_{2} \frac{1}{2}, \frac{3}{p}-1$, but weakly continuous at $t=0$; i.e.,

$$
\lim _{t \rightarrow 0^{+}} \sup _{0<s<t}\langle u(s, \cdot), g(\cdot)\rangle=0 \quad \text { for all } g \in \mathscr{Y} \text { with }\|g\|_{\dot{\mathscr{R}}_{2, p}-\frac{1}{2}, 1-\frac{3}{p}} \leq 1 .
$$

Our main results are stated as follows.

Theorem 1.4. Let $p \in[2,4]$. There exists a positive constant $c$ independent of $\Omega$ such that if $\left\|u_{0}\right\|_{\dot{\mathscr{B}}_{2, p}^{2}, \frac{3}{p}-1} \leq c$, then there exists a unique solution $u \in E_{p}$ of (1-1)
such that

$$
u \in C_{*}\left([0, \infty) ; \dot{\mathscr{B}}_{2}^{\frac{1}{2}, \frac{3}{p}-1}\right) .
$$

Remark 1.5. Due to the inclusion map

$$
H^{\frac{1}{2}} \subseteq \dot{\mathscr{B}}_{2}^{\frac{1}{2}, \frac{3}{p}-1} \text { for } p \geq 2,
$$

Theorem 1.4 is an improvement on Theorem 1.1. The importance of this is that it allows us to construct global solutions of (1-1) for a class of highly oscillating initial velocity $u_{0}$, for example,

$$
u_{0}(x)=\sin \left(\frac{x_{3}}{\varepsilon}\right)\left(-\partial_{2} \phi(x), \partial_{1} \phi(x), 0\right)
$$

where $\phi \in \mathscr{Y}\left(\mathbb{R}^{3}\right)$ and $\varepsilon>0$ is small enough. This type of data is large in the Sobolev norm; however, it is small in the norms of Besov spaces with negative regularity index.

Remark 1.6. As shown in Section 4.2 of [Cannone 2004], for the classical NavierStokes equations (1-2), there exists the following "highly oscillating" initial data: $u_{0}(x) \in \mathscr{S}^{\prime}\left(\mathbb{R}^{3}\right)$ is such that $\hat{u}_{0}(\xi)=0$ if $|\xi| \leq 1 / \varepsilon$. Then

$$
\left\|u_{0}\right\|_{\dot{H}^{1 / 2}} \leq \varepsilon^{1 / 2}\left\|u_{0}\right\|_{\dot{H}^{1}} .
$$

We point out that examples like (1-5) are not included in such initial data. In fact, if $\operatorname{supp} \hat{\phi}(\xi) \subset\{|\xi| \leq 1 / 2 \varepsilon\}$, then the above estimate is satisfied, while if $\hat{\phi}(\xi)$ has no support, it is not sure that (1-6) holds, which implies the norm of $\left\|u_{0}\right\|_{\dot{H}^{1 / 2}}$ may 
not be small enough.

Remark 1.7. The inhomogeneous part of the solution has more regularity:

$$
u-\mathscr{G}(t) u_{0} \in C\left(\mathbb{R}^{+} ; \dot{B}_{2, \infty}^{\frac{1}{2}}\right),
$$

which can be proved by following the proof of Proposition 4.1.

If $u_{0}$ lies in $\dot{H}^{\frac{1}{2}}$, we can obtain the following global well-posedness result.

Theorem 1.8. Let $p \in[2,4]$. There exists a positive constant $c$ independent of $\Omega$ such that, if $u_{0}$ belongs to $\dot{H}^{\frac{1}{2}}$ with $\left\|u_{0}\right\|_{\dot{\mathscr{S}}_{2}, \frac{1}{2}, \frac{3}{p}-1} \leq c$, then there exists a unique global solution of (1-1) in $C\left(\mathbb{R}^{+}, \dot{H}^{\frac{1}{2}}\right)$.

Remark 1.9. Since we only impose the smallness condition of the initial data in the norm of $\mathscr{\Re}_{2}^{\frac{1}{2}, p}, \frac{3}{p}-1$, this allows us to obtain the global well-posedness of (1-1) for a class of highly oscillating initial velocity $u_{0}$. Moreover, the uniqueness holds in the class $C\left(\mathbb{R}^{+}, \dot{H}^{\frac{1}{2}}\right)$; i.e., it is unconditional.

The structure of this paper is as follows. In Section 2, we recall some basic facts about Littlewood-Paley theory and the functional spaces. In Section 3, we recall some results concerning the Stokes-Coriolis semigroup's regularizing effect. Section 4 is devoted to the important bilinear estimates. In Section 5, we prove Theorem 1.4 and Theorem 1.8.

\section{Littlewood-Paley theory and the function spaces}

First of all, we introduce the Littlewood-Paley decomposition. Choose two radial functions $\varphi, \chi \in \mathscr{T}\left(\mathbb{R}^{3}\right)$ supported in $\mathscr{C}=\left\{\xi \in \mathbb{R}^{3}, \frac{3}{4} \leq|\xi| \leq \frac{8}{3}\right\}, \mathscr{B}=\left\{\xi \in \mathbb{R}^{3},|\xi| \leq \frac{4}{3}\right\}$, respectively, such that

$$
\sum_{j \in \mathbb{Z}} \varphi\left(2^{-j} \xi\right)=1 \quad \text { for all } \xi \neq 0
$$

For $f \in \mathscr{S}^{\prime}\left(\mathbb{R}^{3}\right)$, the frequency localization operators $\Delta_{j}$ and $S_{j}(j \in \mathbb{Z})$ are defined by

$$
\Delta_{j} f=\varphi\left(2^{-j} D\right) f, \quad S_{j} f=\chi\left(2^{-j} D\right) f, \quad D=\frac{\nabla_{x}}{i} .
$$

Moreover, we have

$$
S_{j} f=\sum_{k=-\infty}^{j-1} \Delta_{k} f \quad \text { in } \mathscr{E}^{\prime}\left(\mathbb{R}^{3}\right) .
$$

Here we denote by $\mathscr{L}^{\prime}\left(\mathbb{R}^{3}\right)$ the dual space of

$$
\mathscr{L}\left(\mathbb{R}^{3}\right)=\left\{f \in \mathscr{S}\left(\mathbb{R}^{3}\right): D^{\alpha} \hat{f}(0)=0 \text { for all multiindices } \alpha \in(\mathbb{N} \cup 0)^{3}\right\} .
$$


With our choice of $\varphi$, it is easy to verify that
$\Delta_{j} \Delta_{k} f=0 \quad$ if $|j-k| \geq 2$
and
$\Delta_{j}\left(S_{k-1} f \Delta_{k} f\right)=0$
if $|j-k| \geq 5$.

In the sequel, we will constantly use Bony's decomposition [1981]:

$$
f g=T_{f} g+T_{g} f+R(f, g),
$$

with

$$
T_{f} g=\sum_{j \in \mathbb{Z}} S_{j-1} f \Delta_{j} g, \quad R(f, g)=\sum_{j \in \mathbb{Z}} \Delta_{j} f \widetilde{\Delta}_{j} g, \quad \tilde{\Delta}_{j} g=\sum_{\left|j^{\prime}-j\right| \leq 1} \Delta_{j^{\prime}} g .
$$

Definition 2.1 (homogeneous Besov space). Let $s \in \mathbb{R}, 1 \leq p, q \leq+\infty$. The homogeneous Besov space $\dot{B}_{p, q}^{s}$ is defined by

$$
\dot{B}_{p, q}^{s}:=\left\{f \in \mathscr{E}^{\prime}\left(\mathbb{R}^{3}\right):\|f\|_{\dot{B}_{p, q}^{s}}<+\infty\right\},
$$

where

$$
\|f\|_{\dot{B}_{p, q}^{s}}:=\left\|2^{k s}\right\| \Delta_{k} f\left\|_{L^{p}}\right\|_{l^{q}} .
$$

If $p=q=2, \dot{B}_{2,2}^{s}$ is equivalent to the homogeneous Sobolev space $\dot{H}^{s}$.

Definition 2.2 (hybrid-Besov space). Let $s, \sigma \in \mathbb{R}, 1 \leq p \leq+\infty$. The hybrid-Besov space $\dot{\mathscr{R}}_{2, p}^{s, \sigma}$ is defined by

$$
\dot{\mathscr{S}}_{2, p}^{s, \sigma}:=\left\{f \in \mathscr{L}^{\prime}\left(\mathbb{R}^{3}\right):\|f\|_{\dot{\mathscr{P}}_{2, p}^{s, \sigma}}<+\infty\right\},
$$

where

$$
\|f\|_{\dot{\beta}_{2, p}^{s, \sigma}}:=\sup _{2^{k} \leq \Omega} 2^{k s}\left\|\Delta_{k} f\right\|_{L^{2}}+\sup _{2^{k}>\Omega} 2^{k \sigma}\left\|\Delta_{k} f\right\|_{L^{p}} .
$$

The norm of the space $\widetilde{L}_{T}^{r}\left(\dot{\mathscr{B}}_{2, p}^{s, \sigma}\right)$ is defined by

$$
\|f\|_{\tilde{L}_{T}^{r}\left(\dot{\Re}_{2, p}^{s, \sigma}\right)}:=\sup _{2^{k} \leq \Omega} 2^{k s}\left\|\Delta_{k} f\right\|_{L_{T}^{r} L^{2}}+\sup _{2^{k}>\Omega} 2^{k \sigma}\left\|\Delta_{k} f\right\|_{L_{T}^{r} L^{p}} .
$$

It is easy to check that $L_{T}^{r}\left(\dot{\mathscr{B}}_{2, p}^{s, \sigma}\right) \subseteq \widetilde{L}_{T}^{r}\left(\dot{\mathscr{\Re}}_{2, p}^{s, \sigma}\right)$, where the norm of $L_{T}^{r}\left(\dot{\mathscr{B}}_{2, p}^{s, \sigma}\right)$ is defined by

$$
\|f\|_{L_{T}^{r}\left(\dot{\Re}_{2, p}^{s, \sigma}\right)}:=\|\| f(t)\left\|_{\dot{\mathscr{B}}_{2, p}^{s, \sigma}}\right\|_{L_{T}^{r}} .
$$

Bernstein's lemma will be repeatedly used throughout this paper:

Lemma 2.3 [Chemin 1995]. Let $1 \leq p \leq q \leq+\infty$. Then for any $\beta, \gamma \in(\mathbb{N} \cup\{0\})^{3}$, there exists a constant $C$ independent of $f, j$ such that, for any $f \in L^{p}$,

$$
\begin{gathered}
\operatorname{supp} \hat{f} \subseteq\left\{|\xi| \leq A_{0} 2^{j}\right\} \Rightarrow\left\|\partial^{\gamma} f\right\|_{L^{q}} \leq C 2^{j|\gamma|+j n\left(\frac{1}{p}-\frac{1}{q}\right)}\|f\|_{L^{p}}, \\
\operatorname{supp} \hat{f} \subseteq\left\{A_{1} 2^{j} \leq|\xi| \leq A_{2} 2^{j}\right\} \Rightarrow\|f\|_{L^{p}} \leq C 2^{-j|\gamma|} \sup _{|\beta|=|\gamma|}\left\|\partial^{\beta} f\right\|_{L^{p}} .
\end{gathered}
$$


Proposition 2.4. Let $\phi \in \mathscr{Y}\left(\mathbb{R}^{3}\right)$ and $p>3$. If $\phi_{\varepsilon}(x):=e^{i \frac{x_{1}}{\varepsilon}} \phi(x)$, then, for any $0<\varepsilon \leq \Omega^{-1}$,

$$
\left\|\phi_{\varepsilon}\right\|_{\dot{\mathscr{B}}_{2} \frac{1}{2}, \frac{3}{p}-1} \leq C \varepsilon^{1-\frac{3}{p}},
$$

where $C$ is a constant independent of $\varepsilon$.

Proof. Let $j_{0} \in \mathbb{N}$ be such that $\Omega \leq 2^{j_{0}} \sim \varepsilon^{-1}$. By Lemma 2.3, we have

$$
\sup _{j \geq j_{0}} 2^{\left(\frac{3}{p}-1\right) j}\left\|\Delta_{j} \phi_{\varepsilon}\right\|_{L^{p}} \leq C 2^{\left(\frac{3}{p}-1\right) j_{0}} \leq C \varepsilon^{1-\frac{3}{p}} .
$$

Noting that $e^{i \frac{x_{1}}{\varepsilon}}=\left(-i \varepsilon \partial_{1}\right)^{N} e^{i \frac{x_{1}}{\varepsilon}}$ for any $N \in \mathbb{N}$, we get, by integration by parts, $\Delta_{j} \phi_{\varepsilon}(x)=(i \varepsilon)^{N} 2^{3 j} \int_{\mathbb{R}^{3}} e^{i \frac{y_{1}}{\varepsilon}} \partial_{y_{1}}^{N}\left(h\left(2^{j}(x-y)\right) \phi(y)\right) d y, \quad h(x):=\left(\mathscr{F}^{-1} \varphi\right)(x)$.

By the Leibnitz formula, we have

$$
\left|\Delta_{j} \phi_{\varepsilon}(x)\right| \leq C \varepsilon^{N} 2^{3 j} \sum_{k=0}^{N} 2^{k j} \int_{\mathbb{R}^{3}}\left|\left(\partial_{y_{1}}^{k} h\right)\left(2^{j}(x-y)\right)\right|\left|\partial_{y_{1}}^{N-k} \phi(y)\right| d y,
$$

from which, along with Young's inequality, we infer that, for $j \geq 0$,

$$
\left\|\Delta_{j} \phi_{\varepsilon}\right\|_{L^{q}} \leq C \varepsilon^{N} \sum_{k=0}^{N} 2^{k j} 2^{3 j}\left\|\left(\partial_{y_{1}}^{k} h\right)\left(2^{j} y\right)\right\|_{L^{1}}\left\|\partial_{y_{1}}^{N-k} \phi(y)\right\|_{L^{q}} \leq C \varepsilon^{N_{2} 2^{j N}},
$$

and for $j \leq 0$,

$\left\|\Delta_{j} \phi_{\varepsilon}\right\|_{L^{q}} \leq C \varepsilon^{N} \sum_{k=0}^{N} 2^{k j} 2^{3 j}\left\|\left(\partial_{y_{1}}^{k} h\right)\left(2^{j} y\right)\right\|_{L^{q}}\left\|\partial_{y_{1}}^{N-k} \phi(y)\right\|_{L^{1}} \leq C \varepsilon^{N} 2^{\left(1-\frac{1}{q}\right) 3 j}$.

Thus we have

$$
\begin{gathered}
\sup _{\Omega<2^{j}<2^{j_{0}}} 2^{\left(\frac{3}{p}-1\right) j}\left\|\Delta_{j} \phi_{\varepsilon}\right\|_{L^{p}} \leq C \varepsilon^{N} 2^{\left(N-1+\frac{3}{p}\right) j_{0}} \leq C \varepsilon^{1-\frac{3}{p}}, \\
\sup _{2^{j} \leq \Omega} 2^{\frac{j}{2}}\left\|\Delta_{j} \phi_{\varepsilon}\right\|_{L^{2}} \leq C \Omega^{\frac{1}{2}} \varepsilon^{N} \leq C \varepsilon^{N-\frac{1}{2}} .
\end{gathered}
$$

Summing up the above estimates yields that

$$
\left\|\phi_{\varepsilon}\right\|_{\dot{\mathscr{B}}_{2}^{2}, \frac{1}{p}, \frac{3}{p}-1} \leq C \varepsilon^{1-\frac{3}{p}} .
$$

The proof of Proposition 2.4 is completed. 


\section{Regularizing effect of the Stokes-Coriolis semigroup}

We consider the linear system

$$
\left\{\begin{array}{l}
u_{t}-v \Delta u+\Omega e_{3} \times u+\nabla p=0, \\
\operatorname{div} u=0 \\
u(0, x)=u_{0}(x)
\end{array}\right.
$$

From [Giga et al. 2005; Hieber and Shibata 2010, Proposition 2.1], we know that

$$
\hat{u}(t, \xi)=\cos \left(\Omega \frac{\xi_{3}}{|\xi|} t\right) e^{-v|\xi|^{2} t} I \widehat{u_{0}}(\xi)+\sin \left(\Omega \frac{\xi_{3}}{|\xi|} t\right) e^{-v|\xi|^{2} t} R(\xi) \widehat{u_{0}}(\xi),
$$

for $t \geq 0$ and $\xi \in \mathbb{R}^{3}$, where $I$ is the identity matrix and

$$
R(\xi)=\left(\begin{array}{ccc}
0 & \xi_{3} /|\xi| & -\xi_{2} /|\xi| \\
-\xi_{3} /|\xi| & 0 & \xi_{1} /|\xi| \\
\xi_{2} /|\xi| & -\xi_{1} /|\xi| & 0
\end{array}\right) .
$$

The Stokes-Coriolis semigroup is explicitly represented by

$$
\mathscr{G}(t) f=\left[\cos \left(\Omega R_{3} t\right) I+\sin \left(\Omega R_{3} t\right) R\right] e^{\nu t \Delta} f, \quad \text { for } t \geq 0, f \in L_{\sigma}^{p},
$$

where $\widehat{R_{3} f}(\xi):=\left(\xi_{3} /|\xi|\right) \hat{f}(\xi)$ for $\xi \neq 0$.

Proposition 3.1 (smoothing effect of the Stokes-Coriolis semigroup). Let $\mathscr{b}$ be a ring centered at 0 in $\mathbb{R}^{3}$. Then there exist positive constants $c$ and $C$ depending only on $v$ such that if supp $\hat{u} \subset \lambda \mathscr{C}$, then we have:

(i) for any $\lambda>0$,

$$
\|\mathscr{G}(t) u\|_{L^{2}} \leq C e^{-c \lambda^{2} t}\|u\|_{L^{2}}
$$

(ii) if $\lambda \gtrsim \Omega$, then, for any $1 \leq p \leq \infty$,

$$
\|\mathscr{G}(t) u\|_{L^{p}} \leq C e^{-c \lambda^{2} t}\|u\|_{L^{p}} .
$$

Proof. (i) Thanks to (3-2) and the Plancherel theorem, we get

$$
\|\mathscr{G}(t) u\|_{L^{2}}=\|\hat{\mathscr{G}}(t, \xi) \hat{u}(\xi)\|_{L^{2}} \leq C\left\|e^{-v|\xi|^{2} t} \hat{u}(\xi)\right\|_{2} \leq C e^{-v \lambda^{2} t}\|u\|_{2},
$$

where we have used the support property of $\hat{u}(\xi)$.

(ii) Let $\phi \in \mathscr{D}\left(\mathbb{R}^{3} \backslash\{0\}\right)$, which equals 1 near the ring $\mathscr{C}$. Set

$$
g(t, x):=(2 \pi)^{-3} \int_{\mathbb{R}^{3}} e^{i x \cdot \xi} \phi\left(\lambda^{-1} \xi\right) \widehat{\mathscr{G}}(t, \xi) d \xi .
$$

To prove (3-5), it suffices to show

$$
\|g(x, t)\|_{L^{1}} \leq C e^{-c \lambda^{2} t} .
$$


Thanks to (3-3), we infer that

$$
\int_{|x| \leq \lambda-1}|g(x, t)| d x \leq C \int_{|x| \leq \lambda-1} \int_{\mathbb{R}^{3}}\left|\phi\left(\lambda^{-1} \xi\right)\right||\widehat{\mathscr{G}}(t, \xi)| d \xi d x \leq C e^{-c \lambda^{2} t} .
$$

Set $L:=x \cdot \nabla_{\xi} /\left(i|x|^{2}\right)$. Noting that $L\left(e^{i x \cdot \xi}\right)=e^{i x \cdot \xi}$, we get, using integration by parts,

$g(x, t)=\int_{\mathbb{R}^{3}} L^{N}\left(e^{i x \cdot \xi}\right) \phi\left(\lambda^{-1} \xi\right) \widehat{\mathscr{G}}(t, \xi) d \xi=\int_{\mathbb{R}^{3}} e^{i x \cdot \xi}\left(L^{*}\right)^{N}\left(\phi\left(\lambda^{-1} \xi\right) \widehat{\mathscr{G}}(t, \xi)\right) d \xi$, where $N \in \mathbb{N}$ is chosen later. Using the Leibnitz formula, it is easy to verify that

$$
\left|\partial^{\gamma}\left(e^{ \pm i \Omega \frac{\xi_{3}}{|\xi|} t}\right)\right| \leq C|\xi|^{-|\gamma|}(1+\Omega t)^{|\gamma|}, \quad\left|\partial^{\gamma}\left(e^{-\nu|\xi|^{2} t}\right)\right| \leq C|\xi|^{-|\gamma|} e^{-\frac{\nu}{2}|\xi|^{2} t} .
$$

Thus we obtain

$$
\begin{aligned}
& \left|\left(L^{*}\right)^{N}\left(\phi\left(\lambda^{-1} \xi\right) \widehat{\mathscr{G}}(t, \xi)\right)\right| \\
& \leq C|x|^{-N} \sum_{\substack{\left|\alpha_{1}\right|+\left|\alpha_{2}\right| \\
+\left|\alpha_{3}\right|=|\alpha| \\
|\alpha| \leq N}} \lambda^{-N+\alpha}\left|\left(\nabla^{N-\alpha} \phi\right)\left(\lambda^{-1} \xi\right) \partial^{\alpha_{1}}\left(e^{ \pm i \Omega \frac{\xi_{3}}{|\xi|} t}\right) \partial^{\alpha_{2}}\left(e^{-v|\xi|^{2} t}\right) \partial^{\alpha_{3}}(I+R(\xi))\right| \\
& \leq C|\lambda x|^{-N} \sum_{\substack{\left|\alpha_{1}\right|+\left|\alpha_{2}\right| \\
+\left|\alpha_{3}\right|=|\alpha| \\
|\alpha| \leq N}} \lambda^{\alpha}\left|\left(\nabla^{N-\alpha} \phi\right)\left(\lambda^{-1} \xi\right)\right||\xi|^{-\left|\alpha_{1}\right|-\left|\alpha_{2}\right|-\left|\alpha_{3}\right|} e^{-\frac{\nu}{2}|\xi|^{2} t}(1+\Omega t)^{\left|\alpha_{1}\right|} .
\end{aligned}
$$

Taking $N=4$, for any $\xi \in\left\{\xi: A^{-1} \lambda \leq|\xi| \leq A \lambda\right\}$ and for some constant $A$ depending on the ring $\mathscr{b}$ and $\lambda \gtrsim \Omega$,

$$
\left|\left(L^{*}\right)^{4}\left(\phi\left(\lambda^{-1} \xi\right) \widehat{\mathscr{G}}(t, \xi)\right)\right| \leq C|\lambda x|^{-4} e^{-\frac{v}{4}|\xi|^{2} t},
$$

which implies that

$$
\int_{|x| \geq \frac{1}{\lambda}}|g(x, t)| d x \leq C e^{-c \lambda^{2} t} \lambda^{3} \int_{|x| \geq \frac{1}{\lambda}}|\lambda x|^{-4} d x \leq C e^{-c \lambda^{2} t},
$$

which, together with (3-7), gives (3-6). Then the inequality (3-5) is proved.

The following proposition is a direct consequence of Proposition 3.1.

Proposition 3.2. Let $s, \sigma \in \mathbb{R}$, and $(p, q) \in[1, \infty]$. Then, for any $u \in \dot{\mathscr{B}}_{2, p}^{s-\frac{2}{q}, \sigma-\frac{2}{q}}$, we have

$$
\|\mathscr{G}(t) u\|_{\widetilde{L}_{T}^{q}\left(\dot{\mathscr{P}}_{2, p}^{s, \sigma}\right)} \leq C\|u\|_{\dot{\mathscr{B}}_{2, p}^{s-\frac{2}{q}}, \sigma-\frac{2}{q}},
$$

and for any $f \in \widetilde{L}_{T}^{1} \mathscr{B}_{2, p}^{s, \sigma}$, we have

$$
\left\|\int_{0}^{t} \mathscr{G}(t-\tau) f(\tau) d \tau\right\|_{\tilde{L}_{T}^{q}\left(\dot{\Re}_{2, p}^{s+\frac{2}{q}, \sigma+\frac{2}{q}}\right)} \leq C\|f(t)\|_{\tilde{L}_{T}^{1}\left(\dot{\mathscr{\Re}}_{2, p}^{s, \sigma}\right)} .
$$


Proof. Here we only prove (3-9). For any $2^{j} \geq \Omega$, we get by Proposition 3.1 that

$$
\left\|\Delta_{j} \int_{0}^{t} \mathscr{G}(t-\tau) f(\tau) d \tau\right\|_{L^{p}} \leq C \int_{0}^{t} e^{-c(t-\tau) 2^{2 j}}\left\|\Delta_{j} f(\tau)\right\|_{L^{p}} d \tau,
$$

from which, along with Young's inequality, it follows that

$$
\begin{aligned}
\left\|\Delta_{j} \int_{0}^{t} \mathscr{G}(t-\tau) f(\tau) d \tau\right\|_{L_{T}^{q} L^{p}} & \leq C\left\|e^{-c t 2^{2 j}}\right\|_{L_{T}^{q}\left\|\Delta_{j} f(\tau)\right\|_{L_{T}^{1} L^{p}}} \leq C 2^{-\frac{2}{q} j}\left\|\Delta_{j} f(\tau)\right\|_{L_{T}^{1} L^{p}} .
\end{aligned}
$$

Similarly, we also have

$$
\begin{aligned}
\left\|\Delta_{j} \int_{0}^{t} \mathscr{G}(t-\tau) f(\tau) d \tau\right\|_{L_{T}^{q} L^{2}} & \leq C\left\|e^{-c t 2^{2 j}}\right\|_{L_{T}^{q}\left\|\Delta_{j} f(\tau)\right\|_{L_{T}^{1} L^{2}}} \leq C 2^{-\frac{2}{q} j}\left\|\Delta_{j} f(\tau)\right\|_{L_{T}^{1} L^{2}} .
\end{aligned}
$$

Then the inequality (3-9) follows from (3-10) and (3-11).

\section{Bilinear estimates}

We study the continuity of the inhomogeneous term in the space $E_{p, T}$ whose norm is defined by

$$
\|u\|_{E_{p, T}}:=\|u\|_{\widetilde{L}^{\infty}\left(0, T ; \dot{B}_{2, p}^{\frac{1}{2}, \frac{3}{p}-1}\right)}+\|u\|_{\widetilde{L}^{1}\left(0, T ; \dot{B_{3}, \frac{5}{2}, \frac{3}{p}+1}\right)} .
$$

We define

$$
B(u, v):=\int_{0}^{t} \mathscr{G}(t-\tau) \mathbb{P} \nabla \cdot(u \otimes v) d \tau,
$$

where $\mathbb{P}$ denotes the Helmholtz projection which is bounded in the $L^{p}$ space for $1<p<\infty$.

Proposition 4.1. Let $p \in[2,4]$. Assume that $u, v \in E_{p, T}$. There exists a constant $C$ independent of $\Omega, u, v$ such that, for any $T>0$,

$$
\|B(u, v)\|_{E_{p, T}} \leq C\|u\|_{E_{p, T}}\|v\|_{E_{p, T}} .
$$

Proof. Thanks to Proposition 3.2, it suffices to show that

$$
\|u v\|_{\widetilde{L}_{T}^{1} \dot{\dot{B}_{2}} \frac{3}{2}, \frac{3}{p}} \leq C\|u\|_{E_{p, T}}\|v\|_{E_{p, T}} .
$$

From Bony's decomposition (2-2) and (2-1), we have

$$
\begin{aligned}
\Delta_{j}(u v) & =\sum_{|k-j| \leq 4} \Delta_{j}\left(S_{k-1} u \Delta_{k} v\right)+\sum_{|k-j| \leq 4} \Delta_{j}\left(S_{k-1} v \Delta_{k} u\right)+\sum_{k \geq j-2} \Delta_{j}\left(\Delta_{k} u \widetilde{\Delta}_{k} v\right) \\
& =: I_{j}+I I_{j}+I I I_{j} .
\end{aligned}
$$


Set $J_{j}:=\left\{\left(k^{\prime}, k\right):|k-j| \leq 4, k^{\prime} \leq k-2\right\}$. Then for $2^{j}>\Omega$,

$$
\begin{aligned}
\left\|I_{j}\right\|_{L_{T}^{1} L^{p}} & \leq \sum_{J_{j}}\left\|\Delta_{j}\left(\Delta_{k^{\prime}} u \Delta_{k} v\right)\right\|_{L_{T}^{1} L^{p}} \\
& \leq\left(\sum_{J_{j, l l}}+\sum_{J_{, l h}}+\sum_{J_{j, h h}}\right)\left\|\Delta_{j}\left(\Delta_{k^{\prime}} u \Delta_{k} v\right)\right\|_{L_{T}^{1} L^{p}}:=I_{j, 1}+I_{j, 2}+I_{j, 3},
\end{aligned}
$$

where

$$
\begin{aligned}
J_{j, l l} & =\left\{\left(k^{\prime}, k\right) \in J_{j}: 2^{k^{\prime}} \leq \Omega, 2^{k} \leq \Omega\right\}, \\
J_{j, l h} & =\left\{\left(k^{\prime}, k\right) \in J_{j}: 2^{k^{\prime}} \leq \Omega, 2^{k}>\Omega\right\}, \\
J_{j, h h} & =\left\{\left(k^{\prime}, k\right) \in J_{j}: 2^{k^{\prime}}>\Omega, 2^{k}>\Omega\right\} .
\end{aligned}
$$

We get by using Lemma 2.3 that

$$
\begin{aligned}
& I_{j, 1} \leq C \sum_{\left(k^{\prime}, k\right) \in J_{j, l l}}\left\|\Delta_{k^{\prime}} u\right\|_{L_{T}^{\infty} L^{\infty} 2^{k\left(\frac{3}{2}-\frac{3}{p}\right)}}\left\|\Delta_{k} v\right\|_{L_{T}^{1} L^{2}} \\
& \leq C \sum_{\left(k^{\prime}, k\right) \in J_{j, l l}} 2^{{\frac{k^{\prime}}{2}}^{\prime}}\left\|\Delta_{k^{\prime}} u\right\|_{L_{T}^{\infty} L^{2}} 2^{k^{\prime}}\left\|\Delta_{k} v\right\|_{L_{T}^{1} L^{2}} 2^{k\left(\frac{3}{2}-\frac{3}{p}\right)} \\
& \leq C\|u\|_{\tilde{L}_{T}^{\infty} \dot{\mathscr{g}} \frac{1}{2, p}, \frac{3}{p}-1}\|v\|_{\widetilde{L}_{T}^{1} \frac{\dot{\beta}_{2}, \frac{5}{2}, \frac{3}{p}+1}{}} \sum_{\left(k^{\prime}, k\right) \in J_{j, l l}} 2^{\left(k^{\prime}-k\right)} 2^{-\frac{3}{p} k} \\
& \leq C 2^{-\frac{3}{p} j}\|u\|_{\widetilde{L}_{T}^{\infty} \dot{\dot{\beta}_{2}} \frac{1}{2}, \frac{3}{p}-1}\|v\|_{\widetilde{L}_{T}^{1} \dot{\mathscr{\beta}}_{2, p}^{\frac{5}{2}, \frac{3}{p}}+1},
\end{aligned}
$$

where we used in the last inequality the fact that

$$
\sum_{\left(k^{\prime}, k\right) \in J_{j, l l}} 2^{\left(k^{\prime}-k\right)} 2^{-\frac{3}{p} k} \leq \sum_{k^{\prime} \leq k-2} 2^{\left(k^{\prime}-k\right)} \sum_{|k-j| \leq 4} 2^{-\frac{3}{p} k} \leq C 2^{-\frac{3}{p} j},
$$

with $C$ independent of $j$. Similarly, we have

$$
\begin{aligned}
I_{j, 2} & \leq \sum_{\left(k^{\prime}, k\right) \in J_{j, l h}}\left\|\Delta_{k^{\prime}} u\right\|_{L_{T}^{\infty} L^{\infty}}\left\|\Delta_{k} v\right\|_{L_{T}^{1} L^{p}} \\
& \leq C \sum_{\left(k^{\prime}, k\right) \in J_{j, l h}} 2^{\frac{k^{\prime}}{2}}\left\|\Delta_{k^{\prime}} u\right\|_{L_{T}^{\infty} L^{2}} 2^{k^{\prime}}\left\|\Delta_{k} v\right\|_{L_{T}^{1} L^{p}} \\
& \leq C 2^{-\frac{3}{p} j}\|u\|_{\widetilde{L}_{T}^{\infty} \dot{\mathfrak{S}_{2}, \frac{1}{2}, \frac{3}{p}-1}}\|v\|_{\widetilde{L}_{T}^{1} \dot{\mathscr{S}}_{2, p}^{\frac{5}{2}, \frac{3}{p}+1}}
\end{aligned}
$$


and

$$
\begin{aligned}
I_{j, 3} & \leq \sum_{\left(k^{\prime}, k\right) \in J_{j, h h}}\left\|\Delta_{k^{\prime}} u\right\|_{L_{T}^{\infty} L^{\infty}}\left\|\Delta_{k} v\right\|_{L_{T}^{1} L^{p}} \\
& \leq C \sum_{\left(k^{\prime}, k\right) \in J_{j, h h}} 2^{k^{\prime}\left(\frac{3}{p}-1\right)}\left\|\Delta_{k^{\prime}} u\right\|_{L_{T}^{\infty} L^{p}} 2^{k^{\prime}}\left\|\Delta_{k} v\right\|_{L_{T}^{1} L^{p}} \\
& \leq C 2^{-\frac{3}{p} j}\|u\|_{\tilde{L}_{T}^{\infty} \dot{\mathscr{B}}_{2, p}^{\frac{1}{2}, \frac{3}{p}-1}}\|v\|_{\widetilde{L}_{T^{1}}^{1} \dot{\mathscr{S}}_{2, p}^{\frac{5}{2}, \frac{3}{p}+1}} .
\end{aligned}
$$

On the other hand, for $2^{j} \leq \Omega$, we have

$$
\begin{aligned}
\left\|I_{j}\right\|_{L_{T}^{1} L^{2}} & \leq \sum_{J_{j}}\left\|\Delta_{j}\left(\Delta_{k^{\prime}} u \Delta_{k} v\right)\right\|_{L_{T}^{1} L^{2}} \\
& \leq\left(\sum_{J_{j, l l}}+\sum_{J_{j, l h}}+\sum_{J_{j, h h}}\right)\left\|\Delta_{j}\left(\Delta_{k^{\prime}} u \Delta_{k} v\right)\right\|_{L_{T}^{1} L^{2}}:=I_{j, 4}+I_{j, 5}+I_{j, 6} .
\end{aligned}
$$

We get by using Lemma 2.3 that

$$
\begin{aligned}
I_{j, 4} & \leq C \sum_{\left(k, k^{\prime}\right) \in J_{j, l l}} 2^{\frac{k^{\prime}}{2}}\left\|\Delta_{k^{\prime}} u\right\|_{L_{T}^{\infty} L^{2}} 2^{k^{\prime}}\left\|\Delta_{k} v\right\|_{L_{T}^{1} L^{2}} \\
& \leq C 2^{-\frac{3 j}{2}}\|u\|_{\tilde{L}_{T}^{\infty} \dot{\mathscr{S}}_{2, p}^{\frac{1}{2}, \frac{3}{p}-1}}\|v\|_{\widetilde{L}_{T}^{1} \dot{\mathscr{S}}_{2, p}^{\frac{5}{2}, \frac{3}{p}+1}},
\end{aligned}
$$

and, noting that $p \leq 4$,

$$
\begin{aligned}
& I_{j, 5} \leq C \sum_{\left(k, k^{\prime}\right) \in J_{j, l h}}\left\|\Delta_{k^{\prime}} u\right\|_{L_{T}^{\infty} L \frac{2 p}{p-2}}\left\|\Delta_{k} v\right\|_{L_{T}^{1} L^{p}} \\
& \leq C \sum_{\left(k, k^{\prime}\right) \in J_{j, l h}} 2^{\frac{k^{\prime}}{2}}\left\|\Delta_{k^{\prime}} u\right\|_{L_{T}^{\infty} L^{2}} 2^{k^{\prime}\left(\frac{3}{p}-\frac{1}{2}\right)}\left\|\Delta_{k} v\right\|_{L_{T}^{1} L^{p}}
\end{aligned}
$$

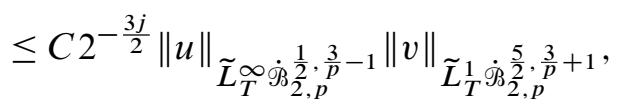

and

$$
\begin{aligned}
I_{j, 6} & \leq C \sum_{\left(k, k^{\prime}\right) \in J_{j, h h}}\left\|\Delta_{k^{\prime}} u\right\|_{L_{T}^{\infty} L_{\frac{2 p}{p-2}}}\left\|\Delta_{k} v\right\|_{L_{T}^{1} L^{p}} \\
& \leq C \sum_{\left(k, k^{\prime}\right) \in J_{j, h h}} 2^{k^{\prime}\left(\frac{3}{p}-1\right)}\left\|\Delta_{k^{\prime}} u\right\|_{L_{T}^{\infty} L^{p}} 2^{k^{\prime}\left(\frac{3}{p}-\frac{1}{2}\right)}\left\|\Delta_{k} v\right\|_{L_{T}^{1} L^{p}} \\
& \leq C 2^{-\frac{3 j}{2}}\|u\|_{\widetilde{L}_{T}^{\infty} \dot{\mathscr{S}}_{2, p}^{\frac{1}{2}, \frac{3}{p}-1}}\|v\|_{\widetilde{L}_{T}^{1} \dot{\mathscr{S}}_{2, p}^{\frac{5}{2}, \frac{3}{p}+1}} .
\end{aligned}
$$

Summing up the estimates for $I_{j, 1}$ through $I_{j, 6}$ yields that

$$
\sup _{2^{j}>1} 2^{j \frac{3}{p}}\left\|I_{j}\right\|_{L_{T}^{1} L^{p}}+\sup _{2^{j} \leq 1} 2^{\frac{3 j}{2}}\left\|I_{j}\right\|_{L_{T}^{1} L^{2}} \leq C\|u\|_{E_{p, T}}\|v\|_{E_{p, T}} .
$$


By the same procedure as the one used to derive (4-3), we have

$$
\sup _{2^{j}>1} 2^{j \frac{3}{p}}\left\|I I_{j}\right\|_{L_{T}^{1} L^{p}}+\sup _{2^{j} \leq 1} 2^{\frac{3 j}{2}}\left\|I I_{j}\right\|_{L_{T}^{1} L^{2}} \leq C\|u\|_{E_{p, T}}\|v\|_{E_{p, T}} .
$$

Set $K_{j}:=\left\{\left(k, k^{\prime}\right): k \geq j-3,\left|k^{\prime}-k\right| \leq 1\right\}$. Then we have

$$
I I I_{j}=\left(\sum_{K_{j, l l}}+\sum_{K_{j, l h}}+\sum_{K_{j, h l}}+\sum_{K_{j, h h}}\right) \Delta_{j}\left(\Delta_{k} u \Delta_{k^{\prime}} v\right):=I I I_{j, 1}+I I I_{j, 2}+I I I_{j, 3}+I I I_{j, 4} \text {, }
$$

where

$$
\begin{aligned}
K_{j, l l} & =\left\{\left(k, k^{\prime}\right) \in K_{j}: 2^{k} \leq \Omega, 2^{k^{\prime}} \leq \Omega\right\}, \\
K_{j, l m} & =\left\{\left(k, k^{\prime}\right) \in K_{j}: 2^{k} \leq \Omega, 2^{k^{\prime}}>\Omega\right\}, \\
K_{j, h m} & =\left\{\left(k, k^{\prime}\right) \in K_{j}: 2^{k}>\Omega, 2^{k^{\prime}} \leq \Omega\right\}, \\
K_{j, h h} & =\left\{\left(k, k^{\prime}\right) \in K_{j}: 2^{k}>\Omega, 2^{k^{\prime}}>\Omega\right\} .
\end{aligned}
$$

We get by Lemma 2.3 that

$$
\begin{aligned}
& \left\|I I I_{j, 1}\right\|_{L_{T}^{1} L^{p}} \leq C 2^{3 j\left(1-\frac{1}{p}\right)} \sum_{\left(k, k^{\prime}\right) \in K_{j, l l}}\left\|\Delta_{k} u \Delta_{k^{\prime}} v\right\|_{L_{T}^{1} L^{1}} \\
& \leq C 2^{3 j\left(1-\frac{1}{p}\right)} \sum_{\left(k, k^{\prime}\right) \in K_{j, l l}} 2^{\frac{k}{2}}\left\|\Delta_{k} u\right\|_{L_{T}^{\infty} L^{2}} 2^{-\frac{k}{2}} 2^{k^{\prime} \frac{5}{2}}\left\|\Delta_{k^{\prime}} v\right\|_{L_{T}^{1} L^{2}} 2^{-k^{\prime} \frac{5}{2}} \\
& \leq C 2^{3 j\left(1-\frac{1}{p}\right)}\|u\|_{\tilde{L}_{T}^{\infty} \dot{\mathscr{B}}_{2, p}^{\frac{1}{2}, \frac{3}{p}-1}}\|v\|_{\widetilde{L}_{T}^{1} \dot{\mathscr{B}}_{2, p}^{\frac{5}{2}, \frac{3}{p}+1}} \sum_{\left(k, k^{\prime}\right) \in K_{j, l l}} 2^{-\frac{k}{2}-\frac{5}{2} k^{\prime}}
\end{aligned}
$$

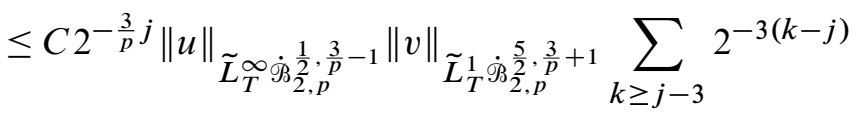

$$
\begin{aligned}
& \leq C 2^{-\frac{3}{p} j}\|u\|_{E_{p, T}}\|v\|_{E_{p, T}} \text {, }
\end{aligned}
$$

and

$$
\left\|I I I_{j, 1}\right\|_{L_{T}^{1} L^{2}} \leq C 2^{\frac{3 j}{2}} \sum_{\left(k, k^{\prime}\right) \in K_{j, l l}}\left\|\Delta_{k} u \Delta_{k^{\prime}} v\right\|_{L_{T}^{1} L^{1}} \leq C 2^{-\frac{3 j}{2}}\|u\|_{E_{p, T}}\|v\|_{E_{p, T}} .
$$

Similarly, we obtain

$$
\begin{aligned}
& \left\|I I I_{j, 2}+I I I_{j, 3}\right\|_{L_{T}^{1} L^{p}} \\
& \quad \leq C 2^{\frac{3 j}{2}} \sum_{\left(k, k^{\prime}\right) \in K_{j, l h} \cup K_{j, h l}}\left\|\Delta_{k} u \Delta_{k^{\prime}} v\right\|_{L_{T}^{1} L^{\frac{2 p}{2+p}}} \\
& \quad \leq C 2^{\frac{3 j}{2}}\left(\sum_{K_{j, l h}}\left\|\Delta_{k} u\right\|_{L_{T}^{\infty} L^{2}}\left\|\Delta_{k^{\prime}} v\right\|_{L_{T}^{1} L^{p}}+\sum_{K_{j, h l}}\left\|\Delta_{k} u\right\|_{L_{T}^{1} L^{p}}\left\|\Delta_{k^{\prime}} v\right\|_{L_{T}^{\infty} L^{2}}\right) \\
& \quad \leq C 2^{-\frac{3}{p} j\|u\|_{E_{, T}}\|v\|_{E_{p, T}}}
\end{aligned}
$$


and

$$
\begin{aligned}
\left\|I I I_{j, 2}+I I I_{j, 3}\right\|_{L_{T}^{1} L^{2}} & \leq C 2^{\frac{3}{p} j} \sum_{\left(k, k^{\prime}\right) \in K_{j, l} \cup K_{j, h l}}\left\|\Delta_{k} u \Delta_{k^{\prime}} v\right\|_{L_{T}^{1} L \frac{2 p}{2+p}} \\
& \leq C 2^{-\frac{3 j}{2}}\|u\|_{E_{p, T}}\|v\|_{E_{p, T}} .
\end{aligned}
$$

Finally, due to $2 \leq p \leq 4$, we have

$$
\begin{aligned}
\left\|I I I_{j, 4}\right\|_{L_{T}^{1} L^{p}} & \leq C 2^{\frac{3}{p} j} \sum_{\left(k, k^{\prime}\right) \in K_{j, h h}}\left\|\Delta_{k} u \Delta_{k^{\prime}} v\right\|_{L_{T}^{1} L^{\frac{p}{2}}} \\
& \leq C 2^{\frac{3}{p} j} \sum_{\left(k, k^{\prime}\right) \in K_{j, h h}}\left\|\Delta_{k} u\right\|_{L_{T}^{\infty} L^{p}\left\|\Delta_{k^{\prime}} v\right\|_{L_{T}^{1} L^{p}}} \\
& \leq C 2^{-\frac{3}{p} j}\|u\|_{E_{p, T}}\|v\|_{E_{p, T}},
\end{aligned}
$$

and

$\left\|I I I_{j, 4}\right\|_{L_{T}^{1} L^{2}} \leq C 2^{3 j\left(\frac{2}{p}-\frac{1}{2}\right)} \sum_{\left(k, k^{\prime}\right) \in K_{j, h h}}\left\|\Delta_{k} u \Delta_{k^{\prime}} v\right\|_{L_{T}^{1} L^{\frac{p}{2}}} \leq C 2^{-\frac{3 j}{2}}\|u\|_{E_{p, T}}\|v\|_{E_{p, T}}$.

Summing up the estimates of $I I I_{j, 1}-I I I_{j, 4}$, we obtain

$$
\sup _{2^{j}>1} 2^{\frac{3}{p} j}\left\|I I I_{j}\right\|_{L_{T}^{1} L^{p}}+\sup _{2^{j} \leq 1} 2^{\frac{3 j}{2}}\left\|I I I_{j}\right\|_{L_{T}^{1} L^{2}} \leq C\|u\|_{E_{p, T}}\|v\|_{E_{p, T}} .
$$

Then the inequality (4-2) can be deduced from (4-3)-(4-5).

In order to prove the uniqueness of the solution in $C\left(\mathbb{R}^{+} ; \dot{H}^{\frac{1}{2}}\right)$, we establish the following new bilinear estimate in the weighted time-space Besov space introduced in [Chen et al. 2008; 2010b].

Proposition 4.2. Assume that $u, v \in L_{T}^{\infty}\left(\dot{B}_{2, \infty}^{\frac{1}{2}}\right)$. Then, for any $T>0$, we have

$$
\|B(u, v)\|_{L_{T}^{\infty} \dot{B}_{2, \infty}^{\frac{1}{2}}} \leq C\|u\|_{L_{T}^{\infty} \dot{B}_{2, \infty}^{\frac{1}{2}}}\left\|\omega_{j, T^{2}} 2^{\frac{j}{2}}\right\| \Delta_{j} v\left\|_{L_{T}^{\infty} L^{2}}\right\|_{l^{\infty}}
$$

where

$$
\omega_{j, T}:=\sup _{k \geq j} e_{k, T} 2^{\frac{1}{2}(j-k)}, \quad e_{j, T}:=1-e^{-c 2^{2 j} T} .
$$

Remark 4.3. The inequality $e_{j, T} \leq \omega_{j, T}$ (top of page 277) is important to the following estimates. On the other hand, due to the fact $\lim _{T \rightarrow 0} \omega_{j, T}=0$, it can be proved that if $u \in C\left([0, T] ; \dot{H}^{\frac{1}{2}}\right)$, then, for any $\varepsilon>0$, one has

$$
\left\|\omega_{j, T} 2^{\frac{j}{2}}\right\| \Delta_{j} v\left\|_{L_{T}^{\infty} L^{2}}\right\|_{l^{\infty}}<\varepsilon \quad \text { if } T \text { is small enough. }
$$

This point is important in the proof of uniqueness. 
Proof. First we note that $e_{j, T} \leq \omega_{j, T}$ for any $j \in \mathbb{Z}$ and that

$$
\omega_{j, T} \leq 2^{\frac{1}{2}\left(j-j^{\prime}\right)} \omega_{j^{\prime}, T} \quad \text { if } j^{\prime} \leq j, \quad \omega_{j, T} \leq 2 \omega_{j^{\prime}, T} \quad \text { if } j \leq j^{\prime} .
$$

We get by Proposition 3.1 that

$$
\begin{aligned}
\|B(u, v)\|_{\dot{B}_{2, \infty}^{\frac{1}{2}}} & \leq \sup _{j \in \mathbb{Z}} 2^{\frac{j}{2}} \int_{0}^{t}\left\|G(t-\tau) \Delta_{j} \mathbb{P} \nabla \cdot(u \otimes v)\right\|_{L^{2}} d \tau \\
& \leq \sup _{j \in \mathbb{Z}} 2^{\frac{3 j}{2}}\left\|e^{-c 2^{2 j} t}\right\|_{L_{T}^{1}}\left\|\Delta_{j}(u \otimes v)\right\|_{L_{T}^{\infty} L^{2}} \\
& \leq C \sup _{j \in \mathbb{Z}} 2^{-\frac{j}{2}} e_{j, T}\left\|\Delta_{j}(u v)\right\|_{L_{T}^{\infty} L^{2} .}
\end{aligned}
$$

We use Bony's decomposition to estimate $\left\|\Delta_{j}(u v)\right\|_{L_{T}^{\infty} L^{2}}$. Since $e_{j, T} \leq \omega_{j, T}$ and thanks to (4-6), we have

$$
\begin{aligned}
\sum_{|k-j| \leq 4}\left\|\Delta_{j}\left(S_{k-1} u \Delta_{k} v\right)\right\|_{L_{T}^{\infty} L^{2}} & \leq C\|u\|_{L_{T}^{\infty} \dot{B}_{2, \infty}^{\frac{1}{2}}} \sum_{|k-j| \leq 4} 2^{k}\left\|\Delta_{k} v\right\|_{L_{T}^{\infty} L^{2}} \\
& \leq C \omega_{j, T}^{-1} 2^{\frac{j}{2}}\|u\|_{L_{T}^{\infty} \dot{B}_{2, \infty}^{\frac{1}{2}}}\left\|\omega_{k, T} 2^{\frac{k}{2}}\right\| \Delta_{k} v \|_{L_{T}^{\infty} L^{2} \|_{l \infty},}
\end{aligned}
$$

and, again by the same properties of $\omega_{j, T}$,

$$
\begin{aligned}
\left\|S_{k-1} v\right\|_{L^{\infty}} & \leq \sum_{k^{\prime} \leq k-2}\left\|\Delta_{k^{\prime}} v\right\|_{L^{2}} 2^{\frac{3}{2} k^{\prime}} \leq\left\|\omega_{k^{\prime}, T} 2^{\frac{k^{\prime}}{2}}\right\| \Delta_{k^{\prime}} v\left\|_{L_{T}^{\infty} L^{2}}\right\|_{l^{\infty}} \sum_{k^{\prime} \leq k-2} 2^{k^{\prime}} \omega_{k^{\prime}, T}^{-1} \\
& \leq 2^{k} \omega_{k, T}^{-1}\left\|\omega_{k^{\prime}, T} 2^{\frac{k^{\prime}}{2}}\right\| \Delta_{k^{\prime}} v\left\|_{L_{T}^{\infty} L^{2}}\right\|_{l^{\infty}},
\end{aligned}
$$

which implies that

$$
\begin{aligned}
& \sum_{|k-j| \leq 4}\left\|\Delta_{j}\left(S_{k-1} v \Delta_{k} u\right)\right\|_{L_{T}^{\infty} L^{2}} \\
& \quad \leq 2^{\frac{k}{2}} \omega_{k, T}^{-1}\|u\|_{L_{T}^{\infty} \dot{B}_{2, \infty}^{\frac{1}{2}}}\left\|\omega_{k^{\prime}, T} 2^{\frac{k^{\prime}}{2}}\right\| \Delta_{k^{\prime}} v\left\|_{L_{T}^{\infty} L^{2}}\right\|_{l^{\infty}},
\end{aligned}
$$

and for the remainder term,

$$
\begin{aligned}
\sum_{k \geq j-2} \| \Delta_{j}\left(\Delta_{k} u \widetilde{\Delta}_{k} v\right) & \|_{L_{T}^{\infty} L^{2}} \\
& \leq \sum_{k \geq j-2} 2^{\frac{3}{2} j}\left\|\Delta_{j}\left(\Delta_{k} u \widetilde{\Delta}_{k} v\right)\right\|_{L_{T}^{\infty} L^{1}} \\
& \leq C \sum_{k \geq j-2} 2^{\frac{3}{2} j}\left\|\Delta_{k} u\right\|_{L_{T}^{\infty} L^{2}}\left\|\widetilde{\Delta}_{k} v\right\|_{L_{T}^{\infty} L^{2}} \\
& \leq C \omega_{j, T}^{-1} 2^{\frac{j}{2}}\|u\|_{L_{T}^{\infty} \dot{B}_{2, \infty}^{\frac{1}{2}}}\left\|\omega_{k, T} 2^{\frac{k}{2}}\right\| \Delta_{k} v\left\|_{L_{T}^{\infty} L^{2}}\right\|_{l \infty} .
\end{aligned}
$$

Substituting (4-8)-(4-10) into (4-7) concludes the proof. 


\section{Proofs of Theorem 1.4 and Theorem 1.8}

The proof of Theorem 1.4 is based on the following classical lemma.

Lemma 5.1 [Cannone 1995]. Let $X$ be an abstract Banach space and $B: X \times X \rightarrow X$ a bilinear operator, $\|\cdot\|$ being the $X$-norm, such that for any $x_{1} \in X$ and $x_{2} \in X$, we have

$$
\left\|B\left(x_{1}, x_{2}\right)\right\| \leq \eta\left\|x_{1}\right\|\left\|x_{2}\right\| .
$$

Then for any $y \in X$ such that

$$
4 \eta\|y\|<1
$$

the equation

$$
x=y+B(x, x)
$$

has a solution $x$ in $X$. Moreover, this solution $x$ is the only one such that

$$
\|x\| \leq \frac{1-\sqrt{1-4 \eta\|y\|}}{2 \eta} .
$$

Proof of Theorem 1.4. Using the Stokes-Coriolis semigroup, we rewrite the system (1-1) as the integral form

$$
u(x, t)=\mathscr{G}(t) u_{0}-\int_{0}^{t} \mathscr{G}(t-\tau) \mathbb{P} \nabla \cdot(u \otimes u) d \tau:=\mathscr{G}(t) u_{0}+B(u, u) .
$$

Thanks to Proposition 3.2, we have

$$
\left\|\mathscr{G}(t) u_{0}\right\|_{E_{p}} \leq C\left\|u_{0}\right\|_{\dot{\mathscr{B}}_{2, p}^{2}, \frac{3}{p}-1} \leq C c .
$$

Obviously, $B(u, v)$ is bilinear, and we get by Proposition 4.1 that

$$
\|B(u, v)\|_{E_{p}} \leq C\|u\|_{E_{p}}\|v\|_{E_{p}} .
$$

Taking $c$ such that $4 C^{2} c<\frac{3}{4}$, Lemma 5.1 ensures that the equation

$$
u=\mathscr{G}(t) u_{0}+B(u, u)
$$

has a unique solution in the ball $\left\{u \in E_{p}:\|u\|_{E_{p}} \leq \frac{1}{4 C}\right\}$.

Now we prove Theorem 1.8.

Proof of Theorem 1.8. We introduce a Banach space $F_{p}$ whose norm is defined by

$$
\|u\|_{F_{p}}:=\|u\|_{\tilde{L}^{\infty}\left(\mathbb{R}^{+} ; \dot{H}^{\left.\frac{1}{2}\right)}\right.}+\|u\|_{E_{p}} .
$$

Step 1: existence in $F_{p}$. We define the map

$$
\mathscr{T} u:=\mathscr{G}(t) u_{0}+B(u, u) .
$$


Next we prove that, if $c$ is small enough, the map $\mathscr{T}$ has a unique fixed point in the ball

$$
B_{A}:=\left\{u \in F_{p}:\|u\|_{E_{p}} \leq A c,\|u\|_{F_{p}} \leq A\left\|u_{0}\right\|_{\dot{H}^{\frac{1}{2}}}\right\}
$$

for some $A>0$ to be determined later. From Proposition 3.2 and Proposition 4.1, we infer that

$$
\|\mathscr{T} u\|_{E_{p}} \leq C\left\|u_{0}\right\|_{\mathscr{Q}_{2}, \frac{1}{2}, \frac{3}{p}-1}+C\|u\|_{E_{p}}^{2} .
$$

On the other hand, we get by Proposition 3.1 that

$$
\begin{aligned}
\|B(u, u)\|_{\tilde{L}^{\infty}\left(\mathbb{R}^{+} ; \dot{H}^{\frac{1}{2}}\right)} & \\
& \leq\left\|\int_{0}^{t} G(t-\tau) \mathbb{P} \nabla \cdot(u \otimes u)(\tau) d \tau\right\|_{\tilde{L}^{\infty}\left(\mathbb{R}^{+} ; \dot{H} \frac{1}{2}\right)} \\
& \leq C\left(\sum_{j \in \mathbb{Z}} 2^{j}\left(\sup _{t \in \mathbb{R}^{+}} \int_{0}^{t}\left\|G(t-\tau) \Delta_{j} \mathbb{P} \nabla \cdot(u \otimes u)(\tau)\right\|_{L^{2}} d \tau\right)^{2}\right)^{\frac{1}{2}} \\
& \leq C\left\|2^{\frac{3}{2} j} \sup _{t \in \mathbb{R}^{+}} \int_{0}^{t} e^{-\tilde{c} 2^{2 j} t}\right\| \Delta_{j}(u \otimes u)\left\|_{L^{2}} d \tau\right\|_{l^{2}} .
\end{aligned}
$$

In the following, we denote by $\left\{c_{j}\right\}_{j \in \mathbb{Z}}$ a sequence in $l^{2}$ with norm $\left\|\left\{c_{j}\right\}\right\|_{l^{2}(\mathbb{Z})} \leq 1$. We get by Lemma 2.3 that

$$
\begin{aligned}
\sup _{t \in \mathbb{R}^{+}} \int_{0}^{t} e^{-\tilde{c} 2^{2 j}} & \left\|\Delta_{j}\left(T_{u} u\right)\right\|_{L^{2}} d \tau \\
& \leq\left\|e^{-\tilde{c} 2^{2 j} t}\right\|_{L^{1}\left(\mathbb{R}^{+}\right)} \sum_{|k-j| \leq 4}\left\|\Delta_{j}\left(S_{k-1} u \Delta_{k} u\right)\right\|_{L^{\infty}\left(\mathbb{R}^{+} ; L^{2}\right)} \\
& \leq C 2^{-2 j}\left\|S_{k-1} u\right\|_{L^{\infty}\left(\mathbb{R}^{+} ; L^{\infty}\right)} \sum_{|k-j| \leq 4}\left\|\Delta_{k} u\right\|_{L^{\infty}\left(\mathbb{R}^{+} ; L^{2}\right)} \\
& \leq C\|u\|_{\tilde{L}^{\infty}\left(\mathbb{R}^{+} ; \dot{\mathfrak{\beta}}_{2, p}^{\frac{1}{2}, \frac{3}{p}-1}\right)} 2^{k} 2^{-2 j} \sum_{|k-j| \leq 4}\left\|\Delta_{k} u\right\|_{L^{\infty}\left(\mathbb{R}^{+} ; L^{2}\right)} \\
& \leq C 2^{-\frac{3}{2} j}\|u\|_{E_{p}} \sum_{|k-j| \leq 4} 2^{\frac{(k-j)}{2}} 2^{\frac{k}{2}}\left\|\Delta_{k} u\right\|_{L^{\infty}\left(\mathbb{R}^{+} ; L^{2}\right)} \\
& \leq C 2^{-\frac{3}{2} j} c_{j}\|u\|_{E_{p}}\|u\|_{\tilde{L}^{\infty}\left(\mathbb{R}^{+} ; \dot{H} \frac{1}{2}\right)}
\end{aligned}
$$


The remainder term of $u v$ is estimated by

$$
\begin{aligned}
& \sup _{t \in \mathbb{R}^{+}} \int_{0}^{t} e^{-\tilde{c} 2^{2 j} t}\left\|\Delta_{j} R(u, u)\right\|_{L^{2}} d \tau \\
& \leq\left\|e^{-\tilde{c} 2^{2 j} t}\right\|_{L^{\infty}\left(\mathbb{R}^{+}\right)} \sum_{k \geq j-2}\left\|\Delta_{j}\left(\Delta_{k} u \tilde{\Delta}_{k} u\right)\right\|_{L^{1}\left(\mathbb{R}^{+} ; L^{2}\right)} \\
& \leq C \sum_{k \geq j-2}\left\|\tilde{\Delta}_{k} u\right\|_{L^{1}\left(\mathbb{R}^{+} ; L^{\infty}\right)}\left\|\Delta_{k} u\right\|_{L^{\infty}\left(\mathbb{R}^{+} ; L^{2}\right)} \\
& \leq C\|u\|_{\tilde{L}^{1} \dot{\leftrightarrow}_{2, p}^{\frac{5}{2}, \frac{3}{p}+1}} \sum_{k \geq j-2} 2^{-k}\left\|\Delta_{k} u\right\|_{L^{\infty}\left(\mathbb{R}^{+} ; L^{2}\right)} \\
& \leq C\|u\|_{E_{p}} \sum_{k \geq j-2} 2^{-\frac{3}{2} k} 2^{\frac{1}{2} k}\left\|\Delta_{k} u\right\|_{L^{\infty}\left(\mathbb{R}^{+} ; L^{2}\right)} \\
& \leq C 2^{-\frac{3}{2} j} c_{j}\|u\|_{E_{p}}\|u\|_{\widetilde{L}^{\infty}\left(\mathbb{R}^{+} ; \dot{H}^{\frac{1}{2}}\right)} .
\end{aligned}
$$

Combining (5-4)-(5-5) with (5-3) yields that

$$
\|B(u, u)\|_{\widetilde{L}^{\infty}\left(\mathbb{R}^{+} ; \dot{H}^{\frac{1}{2}}\right)} \leq C\|u\|_{E_{p}}\|u\|_{\tilde{L}^{\infty}\left(\mathbb{R}^{+} ; \dot{H}^{\frac{1}{2}}\right)} .
$$

It is easy to verify that

$$
\left\|\mathscr{G}(t) u_{0}\right\|_{\tilde{L}_{T}^{\infty} \dot{H} \frac{1}{2}} \leq C\left\|u_{0}\right\|_{\dot{H} \frac{1}{2}} .
$$

Consequently by (5-2) and the estimate

$$
\left\|u_{0}\right\|_{\dot{\mathscr{P}}_{2} \frac{1}{2}, \frac{3}{p}-1} \leq C\left\|u_{0}\right\|_{\dot{H} \frac{1}{2}}
$$

(which follows from Lemma 2.3 and the definition of the Besov space), we obtain

$$
\|\mathscr{T} u\|_{F_{p}} \leq C\left\|u_{0}\right\|_{\dot{H} \frac{1}{2}}+C\|u\|_{E_{p}}\|u\|_{F_{p}} .
$$

Taking $A=2 C$ and $c>0$ such that $2 C^{2} c \leq \frac{1}{2}$, it follows from (5-2) and (5-6) that the map $\mathscr{T}$ is a map from $B_{A}$ to $B_{A}$. Similarly, it can be proved that $\mathscr{T}$ is also a contraction in $B_{A}$. Thus, the Banach fixed point theorem ensures that the map $\mathscr{T}$ has a unique fixed point in $B_{A}$.

Step 2: uniqueness in $C\left(\mathbb{R}^{+} ; \dot{H}^{\frac{1}{2}}\right)$. Let $u_{1}$ and $u_{2}$ be two solutions of (1-1) in $\left.\overline{C\left(\mathbb{R}^{+}\right.} ; \dot{H}^{\frac{1}{2}}\right)$ with the same initial data $u_{0}$. We consider

$$
\begin{aligned}
u_{1}-u_{2}=B\left(u_{1}-\mathscr{G}(t) u_{0}, u_{1}-u_{2}\right)+B\left(\mathscr{G}(t) u_{0}, u_{1}-u_{2}\right) \\
+B\left(u_{1}-u_{2}, u_{2}-\mathscr{G}(t) u_{0}\right)+B\left(u_{1}-u_{2}, \mathscr{G}(t) u_{0}\right) .
\end{aligned}
$$


Then we get by Proposition 4.2 that

$$
\begin{aligned}
\sup _{t \in[0, T]} & \left\|\left(u_{1}-u_{2}\right)(t)\right\|_{\dot{B}_{2, \infty}^{\frac{1}{2}}} \\
\leq C & \sup _{t \in[0, T]}\left\|\left(u_{1}-u_{2}\right)(t)\right\|_{\dot{B}_{2, \infty}^{\frac{1}{2}}}\left(\left\|\omega_{j, T} 2^{\frac{j}{2}}\right\| \Delta_{j} u_{0}\left\|_{2}\right\|_{l \infty}\right. \\
& \left.\quad+\sup _{t \in[0, T]}\left\|u_{1}(t)-\mathscr{G}(t) u_{0}\right\|_{\dot{H}^{\frac{1}{2}}}+\sup _{t \in[0, T]}\left\|u_{2}(t)-\mathscr{G}(t) u_{0}\right\|_{\dot{H}^{\frac{1}{2}}}\right),
\end{aligned}
$$

where we used the fact $\omega_{j, T} \leq 1$ so that

$$
\left\|\omega_{j, T} 2^{\frac{j}{2}}\right\| \Delta_{j} u\left\|_{L_{T}^{\infty} L^{2}}\right\|_{l^{\infty}} \leq \sup _{t \in[0, T]}\|u(t)\|_{\dot{H}^{\frac{1}{2}}} .
$$

Noticing that $\omega_{j, 0}=0$ and $u_{0} \in \dot{H}^{\frac{1}{2}}$, we have

$$
\left\|\omega_{j, T} 2^{\frac{j}{2}}\right\| \Delta_{j} u_{0}\left\|_{2}\right\|_{l \infty} \leq \frac{1}{3 C}
$$

for $T$ small enough. On the other hand, since $u_{1}, u_{2} \in C\left(\mathbb{R}^{+} ; \dot{H}^{\frac{1}{2}}\right)$, we also have

$$
\sup _{t \in[0, T]}\left\|u_{1}-\mathscr{G}(t) u_{0}\right\|_{\dot{H} \frac{1}{2}}+\sup _{t \in[0, T]}\left\|u_{2}-\mathscr{G}(t) u_{0}\right\|_{\dot{H}^{\frac{1}{2}}} \leq \frac{1}{3 C},
$$

for $T$ small enough. Then (5-7) ensures that $u_{1}(t)=u_{2}(t)$ for $T$ small enough. Then, by a standard continuity argument, we conclude that $u_{1}=u_{2}$ on $[0, \infty)$.

\section{References}

[Babin et al. 1997] A. Babin, A. Mahalov, and B. Nicolaenko, "Regularity and integrability of 3D Euler and Navier-Stokes equations for rotating fluids", Asymptot. Anal. 15:2 (1997), 103-150. MR 99b:76092 Zbl 0890.35109

[Babin et al. 1999] A. Babin, A. Mahalov, and B. Nicolaenko, "Global regularity of 3D rotating Navier-Stokes equations for resonant domains", Indiana Univ. Math. J. 48:3 (1999), 1133-1176. MR 2001b:35241 Zbl 0932.35160

[Bony 1981] J.-M. Bony, "Calcul symbolique et propagation des singularités pour les équations aux dérivées partielles non linéaires”, Ann. Sci. École Norm. Sup. (4) 14:2 (1981), 209-246. MR 84h:35177 Zbl 0495.35024

[Cannone 1995] M. Cannone, Ondelettes, paraproduits et Navier-Stokes, Diderot, Paris, 1995. MR 2000e:35173 Zbl 1049.35517

[Cannone 1997] M. Cannone, "A generalization of a theorem by Kato on Navier-Stokes equations", Rev. Mat. Iberoamericana 13:3 (1997), 515-541. MR 99d:35121 Zbl 0897.35061

[Cannone 2004] M. Cannone, "Harmonic analysis tools for solving the incompressible NavierStokes equations", pp. 161-244 in Handbook of mathematical fluid dynamics, vol. III, edited by S. Friedlander and D. Serre, North-Holland, Amsterdam, 2004. MR 2006c:35216 Zbl 1222.35139

[Cannone et al. 1994] M. Cannone, Y. Meyer, and F. Planchon, "Solutions auto-similaires des équations de Navier-Stokes", Exp. VIII in Séminaire sur les Équations aux Dérivées Partielles, 1993-1994, École Polytech., Palaiseau, 1994. MR 95k:35157 Zbl 0882.35090 
[Chemin 1995] J.-Y. Chemin, Fluides parfaits incompressibles, Astérisque 230, Société Mathématique de France, Paris, 1995. MR 97d:76007 Zbl 0829.76003

[Chemin and Gallagher 2006] J.-Y. Chemin and I. Gallagher, "On the global wellposedness of the 3-D Navier-Stokes equations with large initial data”, Ann. Sci. École Norm. Sup. (4) 39:4 (2006), 679-698. MR 2008h:35265 Zbl 1124.35052

[Chemin and Zhang 2007] J.-Y. Chemin and P. Zhang, "On the global wellposedness to the 3-D incompressible anisotropic Navier-Stokes equations", Comm. Math. Phys. 272:2 (2007), 529-566. MR 2008j:35137 Zbl 1132.35068

[Chemin et al. 2000] J.-Y. Chemin, B. Desjardins, I. Gallagher, and E. Grenier, "Fluids with anisotropic viscosity", M2AN Math. Model. Numer. Anal. 34:2 (2000), 315-335. MR 2001m:35261 Zbl 0954.76012

[Chemin et al. 2006] J.-Y. Chemin, B. Desjardins, I. Gallagher, and E. Grenier, Mathematical geophysics:An introduction to rotating fluids and the Navier-Stokes equations, Oxford Lecture Series in Mathematics and its Applications 32, Oxford University Press, 2006. MR 2007m:76148 Zbl 1205.86001

[Chen et al. 2008] Q. Chen, C. Miao, and Z. Zhang, "On the well-posedness for the viscous shallow water equations", SIAM J. Math. Anal. 40:2 (2008), 443-474. MR 2009j:35265 Zbl 1169.35048

[Chen et al. 2010a] Q. Chen, C. Miao, and Z. Zhang, "Global well-posedness for compressible Navier-Stokes equations with highly oscillating initial velocity", Comm. Pure Appl. Math. 63:9 (2010), 1173-1224. MR 2011e:35045 Zbl 1202.35002

[Chen et al. 2010b] Q. Chen, C. Miao, and Z. Zhang, "Well-posedness in critical spaces for the compressible Navier-Stokes equations with density dependent viscosities", Rev. Mat. Iberoam. 26:3 (2010), 915-946. MR 2012c:35341 Zbl 1205.35189

[Dragičević et al. 2006] O. Dragičević, S. Petermichl, and A. Volberg, "A rotation method which gives linear $L^{p}$ estimates for powers of the Ahlfors-Beurling operator", J. Math. Pures Appl. (9) 86:6 (2006), 492-509. MR 2007k:30074 Zbl 1140.42303

[Fujita and Kato 1964] H. Fujita and T. Kato, "On the Navier-Stokes initial value problem, I", Arch. Rational Mech. Anal. 16 (1964), 269-315. MR 29 \#3774 Zbl 0126.42301

[Giga et al. 2005] Y. Giga, K. Inui, A. Mahalov, and S. Matsui, "Uniform local solvability for the Navier-Stokes equations with the Coriolis force", Methods Appl. Anal. 12:4 (2005), 381-393. MR 2007g:76049 Zbl 1107.76023

[Giga et al. 2006] Y. Giga, K. Inui, A. Mahalov, and S. Matsui, "Navier-Stokes equations in a rotating frame in $\mathbb{R}^{3}$ with initial data nondecreasing at infinity", Hokkaido Math. J. 35:2 (2006), 321-364. MR 2007f:35217 Zbl 1192.35132

[Giga et al. 2007] Y. Giga, K. Inui, A. Mahalov, S. Matsui, and J. Saal, "Rotating Navier-Stokes equations in $\mathbb{R}_{+}^{3}$ with initial data nondecreasing at infinity: the Ekman boundary layer problem", Arch. Ration. Mech. Anal. 186:2 (2007), 177-224. MR 2009i:76176 Zbl 1130.76025

[Giga et al. 2008] Y. Giga, K. Inui, A. Mahalov, and J. Saal, "Uniform global solvability of the rotating Navier-Stokes equations for nondecaying initial data”, Indiana Univ. Math. J. 57:6 (2008), 2775-2791. MR 2010b:35342 Zbl 1159.35055

[Hieber and Shibata 2010] M. Hieber and Y. Shibata, "The Fujita-Kato approach to the NavierStokes equations in the rotational framework", Math. Z. 265:2 (2010), 481-491. MR 2011k:35167 Zbl 1190.35175

[Kato 1984] T. Kato, "Strong $L^{p}$-solutions of the Navier-Stokes equation in $\mathbf{R}^{m}$, with applications to weak solutions", Math. Z. 187:4 (1984), 471-480. MR 86b:35171 Zbl 0545.35073 
[Majda 2003] A. Majda, Introduction to PDEs and waves for the atmosphere and ocean, Courant Lecture Notes in Mathematics 9, Courant Institute of Mathematical Sciences, New York, 2003. MR 2004b:76152 Zbl 01885137

[Pedlosky 1987] J. Pedlosky, Geophysical fluid dynamics, 2nd ed., Springer, New York, 1987. Zbl 0713.76005

Received February 14, 2012. Revised December 18, 2012.

\section{QIONGLEI CHEN}

Institute of ApPlied Physics And Computational Mathematics 100088 BEIJING

\section{CHINA}

chen_qionglei@iapcm.ac.cn

ChANGXing MiaO

Institute of ApPlied Physics And Computational Mathematics 100088 BEIJING

\section{CHINA}

miao_changxing@iapcm.ac.cn

ZHIFEI ZHANG

SCHOOL OF Mathematical SCIENCES

PEKING UNIVERSITY

100871 BEIJING

CHINA

zfzhang@math.pku.edu.cn 


\title{
PACIFIC JOURNAL OF MATHEMATICS
}

\author{
msp.org/pjm
}

Founded in 1951 by E. F. Beckenbach (1906-1982) and F. Wolf (1904-1989)

\section{EDITORS}

V. S. Varadarajan (Managing Editor)

Department of Mathematics

University of California

Los Angeles, CA 90095-1555

pacific@math.ucla.edu

Paul Balmer

Department of Mathematics

University of California

Los Angeles, CA 90095-1555

balmer@math.ucla.edu

Daryl Cooper

Department of Mathematics

University of California

Santa Barbara, CA 93106-3080 cooper@math.ucsb.edu

Jiang-Hua $\mathrm{Lu}$

Department of Mathematics

The University of Hong Kong

Pokfulam Rd., Hong Kong jhlu@maths.hku.hk
Don Blasius

Department of Mathematics University of California

Los Angeles, CA 90095-1555

blasius@math.ucla.edu

Robert Finn

Department of Mathematics Stanford University

Stanford, CA 94305-2125

finn@math.stanford.edu

Sorin Popa

Department of Mathematics

University of California

Los Angeles, CA 90095-1555

popa@math.ucla.edu

Paul Yang

Department of Mathematics

Princeton University

Princeton NJ 08544-1000

yang@math.princeton.edu

\section{PRODUCTION}

Silvio Levy, Scientific Editor, production@msp.org

\section{SUPPORTING INSTITUTIONS}

ACADEMIA SINICA, TAIPEI

CALIFORNIA INST. OF TECHNOLOGY

INST. DE MATEMÁTICA PURA E APLICADA

KEIO UNIVERSITY

MATH. SCIENCES RESEARCH INSTITUTE

NEW MEXICO STATE UNIV.

OREGON STATE UNIV.

\author{
STANFORD UNIVERSITY \\ UNIV. OF BRITISH COLUMBIA \\ UNIV. OF CALIFORNIA, BERKELEY \\ UNIV. OF CALIFORNIA, DAVIS \\ UNIV. OF CALIFORNIA, LOS ANGELES \\ UNIV. OF CALIFORNIA, RIVERSIDE \\ UNIV. OF CALIFORNIA, SAN DIEGO \\ UNIV. OF CALIF., SANTA BARBARA
}

\author{
Vyjayanthi Chari \\ Department of Mathematics \\ University of California \\ Riverside, CA 92521-0135 \\ chari@math.ucr.edu \\ Kefeng Liu \\ Department of Mathematics \\ University of California \\ Los Angeles, CA 90095-1555 \\ liu@math.ucla.edu \\ Jie Qing \\ Department of Mathematics \\ University of California \\ Santa Cruz, CA 95064 \\ qing@cats.ucsc.edu
}

These supporting institutions contribute to the cost of publication of this Journal, but they are not owners or publishers and have no responsibility for its contents or policies.

See inside back cover or msp.org/pjm for submission instructions.

The subscription price for 2013 is US \$400/year for the electronic version, and \$485/year for print and electronic.

Subscriptions, requests for back issues and changes of subscribers address should be sent to Pacific Journal of Mathematics, P.O. Box 4163, Berkeley, CA 94704-0163, U.S.A. The Pacific Journal of Mathematics is indexed by Mathematical Reviews, Zentralblatt MATH, PASCAL CNRS Index, Referativnyi Zhurnal, Current Mathematical Publications and the Science Citation Index.

The Pacific Journal of Mathematics (ISSN 0030-8730) at the University of California, c/o Department of Mathematics, 798 Evans Hall \#3840, Berkeley, CA 94720-3840, is published monthly except July and August. Periodical rate postage paid at Berkeley, CA 94704, and additional mailing offices. POSTMASTER: send address changes to Pacific Journal of Mathematics, P.O. Box 4163, Berkeley, CA 94704-0163.

PJM peer review and production are managed by EditFLOW ${ }^{\circledR}$ from Mathematical Sciences Publishers.

PUBLISHED BY

mathematical sciences publishers

nonprofit scientific publishing

http://msp.org/

(C) 2013 Mathematical Sciences Publishers 


\section{PACIFIC JOURNAL OF MATHEMATICS}

Volume $262 \quad$ No. $2 \quad$ April 2013

Certifying incompressibility of noninjective surfaces with scl

DANNY CALEGARI

Global well-posedness for the 3D rotating Navier-Stokes equations with highly oscillating initial data

QIONGLEI CHEN, CHANGXING MiAO and ZHIFEI ZHANG

Presenting Schur superalgebras

HOUSSEIN El TURKEY and JONATHAN R. KUJAWA

Classifying zeros of two-sided quaternionic polynomials and computing zeros of two-sided polynomials with complex coefficients

FENG LIANGGUI and ZHAO KAIMING

Coxeter groups, imaginary cones and dominance

XIANG FU

Semicontinuity of automorphism groups of strongly pseudoconvex domains: The low differentiability case

Robert E. Greene, Kang-Tae Kim, Steven G. Krantz and

AERYEONG SEO

Klein four-subgroups of Lie algebra automorphisms

JING-SONG HUANG and JUN YU

Fractal entropy of nonautonomous systems

Rui KuANG, WEN-ChiaO CHENG and BING LI

A GJMS construction for 2-tensors and the second variation of the total

$Q$-curvature

YoshiniKo MaTSUMOTO

Droplet condensation and isoperimetric towers

Matteo Novaga, Andrei Sobolevski and Eugene Stepanov

Brauer's height zero conjecture for metacyclic defect groups

BENJAMIN SAMBALE 